\title{
Aspectos Epidemiológicos do Acidente Ofídico no Vale do Ribeira, São Paulo, 1985 a 1989
}

\section{Epidemiologic Aspects of Snakebites in Vale do Ribeira, São Paulo, 1985-1989}

\author{
Edward G. Martinez'; Maria Cristina T. Vilanova²; \\ Miguel T. Jorge ${ }^{3} \&$ Lindioneza A. Ribeiro ${ }^{3}$
}

\author{
MARTINEZ, E. G.; VILANOVA, M. C. T.; JORGE, M. T. \& RIBEIRO, L. A. Epidemiologic \\ Aspects of Snakebites in Vale do Ribeira, São Paulo, 1985-1989. Cad. Saúde Públ., Rio de \\ Janeiro, 11 (3): 511-515, Jul/Sep, 1995.
}

This study reports on the incidence of snakebites from January 1985 to December 1989 in Vale do Ribeira, São Paulo, Brazil. Overall incidence ranged from 68.7 to 84.2/100,000. There were 840 snakebites during this period, with higher frequency from October to May. The highest incidence rates were observed in the districts of Juguia and Eldorado (159.4 and 131.4/100,000, respectively). Most of the patients were male. Agricultural workers were the most heavily affected. Most of the patients received treatment within 2 hours after the bite. Morbidity was not severe and lethality was $0.2 \%$.

Key words: Snakebites; Epidemiology; Vale do Ribeira

\section{INTRODUÇÃO}

O envenenamento por serpente peçonhenta é uma intoxicação exógena em que o veneno é inoculado no momento da picada. A população sujeita a risco de sofrer estes acidentes está formada principalmente por lavradores (Belluomini et al., 1987; Ribeiro, 1991). Normalmente os acidentes ocorrem nas proximidades das casas e em plantações e, portanto, fora do habitat natural dos ofídios.

No Brasil existem quatro gêneros de serpentes peçonhentas (Bothrops, Crotalus, Lachesis e Micrurus). No Estado de São Paulo não existem, na natureza, serpentes do gênero Lachesis (Hoge \& Romano-Hoge, 1978, 1979).

\footnotetext{
${ }^{I}$ Diretoria Regional do SUS, Secretaria Estadual de Saúde de São Paulo, Rua Pariquera-açu, 41, Registro, SP, 11900-000, Brasil.

${ }^{2}$ Unidade Básica de Saúde IV, Departamento Municipal de Saúde de Registro, Av. Clara Gianotti de Souza, 345, Registro, São Paulo, 11900-000, Brasil.

${ }^{3}$ Departamento de Clínica Médica, Centro de Ciências Biomédicas, Universidade Federal de Uberlândia. Av. Pará, 1.720, Uberlândia, MG, 38400-902, Brasil.
}

Segundo notificações ao Ministério da Saúde, a incidência de acidentes por serpentes peçonhentas no Brasil tem sido, em média, de 15/ 100 mil habitantes. Admite-se, entretanto, devido à existência de subnotificação, que esta incidência possa ser ainda maior (Resende et al., 1989; Ribeiro, 1993).

O Estado de São Paulo apresentou nos anos de 1986, 1987, 1988 e 1989, 8.721 acidentes, com coeficientes de incidência que variaram de 6,8 a 7,4/100 mil habitantes, o que não está dentro das mais altas do país (Ribeiro, 1990; Ribeiro et al., 1993). Sabe-se, entretanto, que a incidência não é, necessariamente, igual em todas as regiões. O Vale do Ribeira é um território situado na região sudeste do estado, limitado ao norte e ao oeste pela Serra de Paranapiacaba, ao sul pelo Estado do Paraná e ao leste pelo Oceano Atlântico. Sua população dedica se prioritariamente à agricultura, ocupação que põe o homem em contato direto com a serpente.

O presente trabalho avalia os acidentes por serpentes peçonhentas registrados nos doze municípios desse Vale, analisando variáveis anotadas nas "fichas de registro de caso". 


\section{MATERIAL E MÉTODO}

No presente trabalho foram avaliados todos os casos de acidentes por serpentes peçonhentas registrados nos doze municípios que fazem parte do "Escritório Regional de Saúde - 49" (ERSA 49), no período de janeiro de 1985 a dezembro de 1989.

Os dados foram obtidos de cada "Ficha de Aplicação de Soros" existente nos arquivos do ERSA 49, na cidade de Registro, desde janeiro de 1985 até julho de 1988. A partir dessa data, os dados passaram a ser obtidos de cada "Ficha Epidemiológica de Acidente por Animal Peçonhento", em uso até a atualidade, existentes nos mesmos arquivos mencionados.
Os dados populacionais de acordo com sexo e faixa etária, para cada município, foram obtidos pelo ERSA 49 com base em estimativas efetuadas a partir do censo de 1980.

\section{RESULTADOS}

Os resultados são apresentados nas Tabelas 1 a 5 e na Figura 1. Acrescenta-se também que: os acidentes ocorreram principalmente na zona rural; de 563 casos em que o dado foi obtido, 441 $(78,3 \%)$ eram lavradores; o quadro clínico do envenenamento foi, quase sempre, leve ou moderado e tendeu a evoluir sem deixar sequielas graves; a letalidade no período estudado foi de $0,2 \%$.

TABELA 1. Incidência de Acidentes por Serpentes, Vale do Ribeira, São Paulo, 1985 a 1989

\begin{tabular}{ccc}
\hline \hline Anos & Número de Casos & Número de Casos por 100 Mil por Ano \\
\hline 1985 & $* 144$ & 68,7 \\
1986 & 162 & 76,3 \\
1987 & 179 & 83,1 \\
1988 & 184 & 84,2 \\
1989 & 171 & 77,0 \\
\hline Total & 840 & - \\
\hline \hline
\end{tabular}

* Média de 168 casos por ano.

TABELA 2. Incidência de Acidentes por Serpentes Segundo Municípios de Ocorrência, Vale do Ribeira, São Paulo, 1985 a 1989

\begin{tabular}{|c|c|c|c|c|c|c|c|c|}
\hline \multirow[b]{2}{*}{ Município } & \multirow[b]{2}{*}{ Casos } & \multirow[b]{2}{*}{$\%$} & \multirow{2}{*}{$\begin{array}{c}\text { Taxa por } \\
100.000 \\
\text { Habitantes }\end{array}$} & \multicolumn{5}{|c|}{ Anos } \\
\hline & & & & 1985 & 1986 & 1987 & 1988 & 1989 \\
\hline Registro & 127 & 15 & 50,4 & 28 & 32 & 21 & 26 & 20 \\
\hline Juquiá & 127 & 15 & 159,4 & 18 & 23 & 33 & 31 & 22 \\
\hline Jacupiranga & 112 & 13 & 57,3 & 16 & 25 & 18 & 22 & 31 \\
\hline Miracatú & 101 & 12 & 105,7 & 25 & 19 & 18 & 15 & 24 \\
\hline Pariquera Açú & 82 & 10 & 121,1 & 9 & 22 & 23 & 14 & 14 \\
\hline Eldorado & 72 & 9 & 131,4 & 10 & 10 & 13 & 22 & 17 \\
\hline Itarirí & 52 & 6 & 99,2 & 4 & 1 & 21 & 17 & 9 \\
\hline Iguape & 49 & 6 & 39,5 & 10 & 10 & 9 & 8 & 12 \\
\hline Sete Barras & 38 & 4 & 62,9 & 7 & 8 & 6 & 8 & 9 \\
\hline Barra do Turvo & 33 & 4 & 119,0 & 7 & 5 & 3 & 9 & 9 \\
\hline Pedro de Toledo & 26 & 3 & 93,1 & 6 & - & 9 & 8 & 3 \\
\hline Cananéia & 11 & 3 & 49,6 & 4 & 7 & 5 & 4 & 1 \\
\hline Total & 840 & 100 & & 144 & 162 & 179 & 184 & 171 \\
\hline
\end{tabular}


TABELA 3. Incidência de Acidentes por Serpentes Segundo Sexo do Paciente, Vale do Ribeira, São Paulo, 1985 a 1989

\begin{tabular}{lrrrrrrr}
\hline \hline & & & \multicolumn{5}{c}{ Anos } \\
\cline { 5 - 8 } Sexo & Casos & $\%$ & 1985 & 1986 & 1987 & 1988 & 1989 \\
\hline Masculino & 663 & 79 & 118 & 124 & 148 & 142 & 131 \\
Feminino & 177 & 21 & 26 & 38 & 31 & 42 & 40 \\
\hline Total & 840 & 100 & 144 & 162 & 179 & 184 & 171 \\
\hline \hline
\end{tabular}

TABELA 4. Incidência de Acidentes por Serpentes Segundo Faixa Etária, Vale do Ribeira, São Paulo, 1985 a 1989

\begin{tabular}{lccc}
\hline \hline Idade & $\begin{array}{c}\text { População na } \\
\text { Metade do Período }\end{array}$ & Casos & $\begin{array}{c}\text { Taxa por } \\
100 \text { mil por Ano }\end{array}$ \\
\hline$<15$ & 70.630 & 189 & 53,5 \\
$15-29$ & 48.763 & 252 & 103,5 \\
$30-44$ & 47.763 & 189 & 80,3 \\
$45-59$ & 21.710 & 147 & $\mathrm{I} 34,5$ \\
$60 \mathrm{e}+$ & 13.414 & 64 & 95,3 \\
\hline \hline
\end{tabular}

TABELA 5. Incidência de Acidentes por Serpentes Segundo o Tempo entre a Picada e o Atendimento Médico, Vale do Ribeira, São Paulo, 1985 a 1989

\begin{tabular}{lcc}
\hline \hline Tempo (horas) & Casos & $\%$ \\
\hline$<2$ & 476 & 57,7 \\
$2+6$ & 255 & 30,4 \\
$6 \mathrm{a}+$ & 66 & 7,9 \\
Ignorado & 43 & 5,0 \\
\hline Total & 840 & 100,0 \\
\hline \hline
\end{tabular}

FIGURA 1. Sazonalidade dos Acidentes Ofídicos no Vale do Ribeira, São Paulo, 1985 a 1989

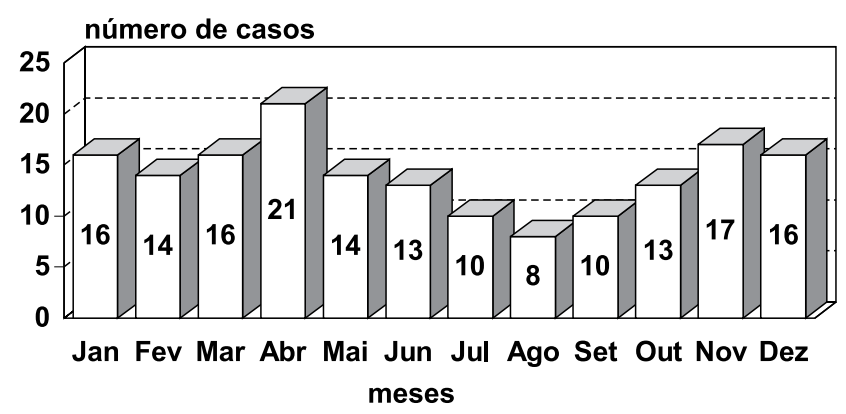




\section{DISCUSSÃO}

Chama a atenção a elevada taxa de incidência de acidentes por serpentes peçonhentas no Vale do Ribeira (68,7 a 84,2 por 100 mil habitantes/ ano), comparada com a incidência no Estado de São Paulo (6,8 a 7,4 por 100 mil habitantes/ano) (Ribeiro, 1990; Ribeiro et al., 1993). As incidências encontradas nos Municípios de Miracatú e Eldorado foram quase 20 vezes superiores às do estado. Fatores responsáveis por esta diferença poderiam ser a concentração populacional em grandes cidades do estado, onde existe pouca possibilidade de contacto com as serpentes e, ao contrário, a distribuição da população do Vale do Ribeira no campo e em pequenas cidades, em região de agricultura pouco mecanizada.

A maior incidência encontrada em pessoas do sexo masculino, coincide com outros dados da literatura (Andrade \& Cardi, 1989; Resende et al., 1989; Ribeiro, 1991: Ribeiro et al., 1993 ).

Embora a faixa etária em que ocorreu o maior número de acidentes tenha sido a de 15 a 29 anos, a maior incidência ocorreu na faixa de 45 a 59 anos. Observa-se também que o grupo de maiores de 60 anos, apesar de apresentar a menor freqüência de acidentes, mostra uma alta incidência. Fenômeno inverso ocorre com o grupo de menores de 15 anos. A distribuição de casos por faixa etária foi semelhante à encontrada em outros estudos (Resende et al., 1989; Ribeiro, 1991; Ribeiro et al., 1993). Não existem dados sobre a incidência por faixa etária em outras regiões do país, para que se possa comparar com os do presente trabalho.

A sazonalidade aqui observada é semelhante à conhecida para a Região Sudeste do país (Resende et al., 1989) e também para o Estado de São Paulo (Ribeiro et al., 1993). A sazonalidade dos acidentes por B. jararaca, serpente responsável pela maioria dos acidentes no estado, é também semelhante, com picos nos meses de março a novembro. Observa-se que os acidentes ocorrem principalmente nos meses de temperatura e pluviosidade elevadas (Ribeiro \& Jorge, 1990), coincidindo com a maior atividade de B. jararaca (Sazima, 1988).

O grupo de trabalhadores mais acometido foi o de lavradores, o que foi também encontrado por Belluomini et al. (1987) e por Ribeiro (1991).
O maior número de casos atendidos antes de haver transcorrido duas horas do acidente, provavelmente se deve a uma distribuição racional dos postos de atendimento a pacientes picados por animais peçonhentos, próximos à área rural (Ribeiro, 1991; Ribeiro et al., 1993), coincidindo com o que acontece em outros municípios com serviços semelhantes (Andrade \& Cardi, 1989). A precocidade do atendimento médico pode ser uma das causas da baixa letalidade encontrada $(0,2 \%)$, semelhante à conhecida para o Estado de São Paulo (0,3\%) (Ribeiro et al., 1993) e menor do que a do País $(0,6 \%)$ (Resende et al., 1989). Em parte ela se explica também pela ocorrência de acidente quase que somente por Bothrops, geralmente $B$. jararaca. Envenenamentos por $B$. jararaca não costumam levar ao óbito (Ribeiro \& Jorge, 1990), ao contrário daquele por Crotalus (Jorge \& Ribeiro, 1994).

\section{RESUMO}

MARTINEZ, E. G.; VILANOVA, M. C. T.; JORGE, M. T. \& RIBEIRO, L. A. Aspectos Epidemiológicos do Acidente Ofídico no Vale do Ribeira, São Paulo, 1985 a 1989. Cad. Saúde Públ., Rio de Janeiro, 11 (3): 511-515, jul/set, 1995.2

O presente trabalho é um estudo da incidência dos acidentes por serpentes peçonhentas nos 12 municípios do Vale do Ribeira, Estado de São Paulo, baseado na avaliação de 840 casos que ocorreram de janeiro de 1985 a dezembro de 1989. A incidência global no Vale mostrou-se muito maior $(68,7$ a $84,2 / 100.000$ habitantes) do que a conhecida para o Estado de São Paulo (6,8 a 7,4/100.000 habitantes). Maior ocorrência foi observada nos primeiros e nos últimos meses do ano, e os municípios de maior incidência foram os de Juquiá (159,4/100.000 habitantes) e Eldorado (131,4/ 100.000 habitantes). As pessoas do sexo masculino foram mais acometidas $(79 \%)$ assim como os lavradores $(52,5 \%)$. A maioria dos casos foi atendida precocemente $(57,7 \%$ 
dentro de duas horas após o acidente). À semelhança do que ocorre em todo o Estado de São Paulo, o quadro clínico provocado pelo envenenamento foi, em geral, leve e a letalidade foi baixa $(0,2 \%)$.

Palavras-Chave: Acidente Ofídico; Epidemiologia; Vale do Ribeira

\section{REFERÊNCIAS BIBLIOGRAFICAS}

ANDRADE, L. R. V. \& CARDI, B. A., 1989. Estrutura Organizacional de um Programa de Prevenção de Acidentes com Animais Peçonhentos. Juiz de Fora, Diretoria Regional de Saúde de Juiz de Fora, Núcleo de Animais Peçonhentos.

BELLUOMINI, H. E.; WAKAMATSU, C. T.; LUCAS, S. M. \& CARDOSO, J. L. C., 1987. Acidentes do trabalho por animais peçonhentos. Revista Brasileira de Saúde Ocupacional, 60: 38-42.

HOGE, A. R. \& ROMANO-HOGE, S. A. R. W. L., 1978/79. Sinopse das serpentes peçonhentas do Brasil. Memórias do Instituto Butantan, 42/43: 373-396.

JORGE, M. T. \& RIBEIRO, L. A., 1994. Epidemiologia e quadro clínico do acidente por cascavel sul-americana (Crotalus durissus). Revista do Instituto de Medicina Tropical de São Paulo, 34: 347-354.
RESENDE, C. C.; ARAÚJO, F. A. A. \& SALLENAVE, R. N. U. R., 1989. Análise Epidemiológica dos Acidentes Ofídicos. Brasília, DF: Ministério da Saúde.

RIBEIRO, L. A., 1990. Epidemiology of ophidic accidents. Memórias do Instituto Butantan, 52 (Supl.): 15-16.

1991. Estudo Epidemiológico de Acidentes por Serpentes Peçonhentas no Estado de São Paulo, Atendidos no Hospital Vital Brazil, 1988. Tese de Mestrado, São Paulo: Faculdade de Saúde Pública, Universidade de São Paulo.

RIBEIRO, L. A. \& JORGE, M. T., 1990. Epidemiologia e quadro clínico dos acidentes por serpentes $B$. jararaca adultas e filhotes. Revista do Instituto de Medicina Tropical de São Paulo, 32: 436-442.

RIBEIRO, L. A.; PIRES DE CAMPOS, V. A. F.; ALBUQUERQUE, M. J. \& TAKAOKA, N. Y., 1993. Acidente ofídico no Estado de São Paulo. Revista da Associação Médica Brasileira, 39: 4-7.

SAZIMA, L, 1988. Um estudo de biologia comportamental da jararaca, Bothrops jararaca, com uso de marcas naturais. Memórias do Instituto Butantan, 50: 83-99. 